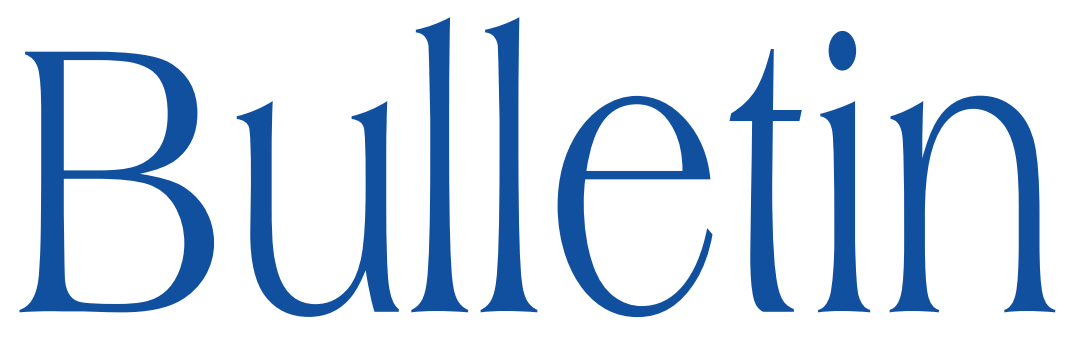

de la SOCIÉTÉ MATHÉMATIQUE DE FRANCE

\title{
SIMILITUDE DES MULTIPLES DES FORMES D'ALBERT EN CARACTÉRISTIQUE 2
}

Detlev W. Hoffmann \& Ahmed Laghribi

\section{Tome 141 Fascicule 2}




\title{
SIMILITUDE DES MULTIPLES DES FORMES D'ALBERT EN CARACTÉRISTIQUE 2
}

\author{
par Detlev W. Hoffmann \& Ahmed Laghribi
}

\begin{abstract}
RÉSumÉ. - Étant donnés $F$ un corps commutatif de caractéristique 2, $\gamma_{1}, \gamma_{2}$ des formes bilinéaires d'Albert et $\pi_{1}, \pi_{2}$ des $k$-formes quadratiques de Pfister, ou $\gamma_{1}, \gamma_{2}$ des $k$-formes bilinéaires de Pfister et $\pi_{1}, \pi_{2}$ des formes quadratiques d'Albert (resp. $\gamma_{1}, \gamma_{2}$ des formes bilinéaires d'Albert et $\pi_{1}, \pi_{2}$ des $k$-formes bilinéaires de Pfister avec la condition que $\gamma_{i} \otimes \pi_{i}, i=1,2$, soient anisotropes), alors on montre que $\gamma_{1} \otimes \pi_{1} \perp$ $\gamma_{2} \otimes \pi_{2} \in I_{q}^{k+3} F\left(\right.$ resp. $\left.I^{k+3} F\right)$ si et seulement si $\gamma_{1} \otimes \pi_{1}$ est semblable à $\gamma_{2} \otimes \pi_{2}$. Un exemple montre que la condition de l'anisotropie est nécessaire dans le cas bilinéaire.

\section{Abstract (Similarity of multiples of Albert forms in characteristic 2)}

Let $F$ be a field of characteristic 2. Let $\gamma_{1}, \gamma_{2}$ be Albert bilinear forms and $\pi_{1}, \pi_{2}$ quadratic $k$-Pfister forms, or $\gamma_{1}, \gamma_{2}$ bilinear $k$-Pfister forms and $\pi_{1}, \pi_{2}$ Albert quadratic forms (resp. $\gamma_{1}, \gamma_{2}$ Albert bilinear forms and $\pi_{1}, \pi_{2}$ bilinear $k$-Pfister forms with the condition that $\gamma_{i} \otimes \pi_{i}, i=1,2$, are anisotropic). Then we show that $\gamma_{1} \otimes \pi_{1} \perp \gamma_{2} \otimes \pi_{2} \in$ $I_{q}^{k+3} F$ (resp. $I^{k+3} F$ ) if and only if $\gamma_{1} \otimes \pi_{1}$ is similar to $\gamma_{2} \otimes \pi_{2}$. We give an example which shows that the anisotropy condition is necessary in the bilinear case.
\end{abstract}

Texte reçu le 7 novembre 2011 et accepté le 30 mars 2012.

Detlev W. Hoffmann, Lehrstuhl VI, Fakultät für Mathematik, Technische Universität Dortmund, 44221 Dortmund, Germany E-mail : detlev.hoffmann@math.tu-dortmund.de

Ahmed Laghribi, Université d'Artois, Faculté des Sciences Jean Perrin, Laboratoire de mathématiques de Lens EA 2462, rue Jean Souvraz - SP18, 62307 Lens, France E-mail : laghribi@euler.univ-artois.fr

Classification mathématique par sujets (2010). - 11E04, 11E81.

Mots clefs. - Formes quadratiques (bilinéaires), formes d'Albert, formes de Pfister, similarité. 


\section{Introduction}

Soit $F$ un corps commutatif. On note $W(F)$ l'anneau de Witt des formes bilinéaires (resp. l'anneau de Witt des formes quadratiques) lorsque $F$ est de caractéristique 2 (resp. lorsque $F$ est de caractéristique différente de 2). Pour tout entier $n \geq 1$, soit $I^{n} F$ la puissance $n$-ième de l'idéal fondamental $I F$ de $W(F)$ (on prend $I^{0} F=W(F)$ ). En caractéristique 2, on note $W_{q}(F)$ le groupe de Witt des formes quadratiques non singulières, et $I_{q}^{n} F$ le groupe $I^{n-1} F \otimes W_{q}(F)$ pour $n \geq 1$, où $\otimes$ est l'action de module de $W(F)$ sur $W_{q}(F)$ [1]. On désigne par $\overline{I^{n}} F$ et $\overline{I_{q}^{n}} F$ les quotients $I^{n} F / I^{n+1} F$ et $I_{q}^{n} F / I_{q}^{n+1} F$, respectivement.

Rappelons qu'une forme quadratique de dimension 6 est dite une forme d'Albert si elle est de discriminant trivial ou d'invariant d'Arf trivial suivant que $F$ est de caractéristique $\neq 2$ ou non. Un résultat bien connu donnant le lien entre ce type de formes quadratiques et les algèbres simples centrales est dû à Jacobson [8], et affirme que deux formes quadratiques d'Albert sont semblables si et seulement si elles ont le même invariant de Clifford. En utilisant le résultat de Merkurjev [17] et celui de Sah [18], on voit que ce résultat de Jacobson équivaut à dire que deux formes quadratiques d'Albert $\gamma_{1}$ et $\gamma_{2}$ sont semblables si et seulement si $\gamma_{1} \perp \gamma_{2} \in I^{3} F$ (ou $\gamma_{1} \perp \gamma_{2} \in I_{q}^{3} F$ si $F$ est de caractéristique 2). C'est ce que Mammone et Shapiro ont utilisé dans [16] pour donner une autre preuve de ce résultat de Jacobson. Plus tard, en caractéristique $\neq 2$, le premier auteur a répondu par l'affirmative a une conjecture d'Izhboldin généralisant le résultat de Jacobson, et affirmant que si $\pi_{1}, \pi_{2}$ sont des $k$-formes quadratiques de Pfister et $\gamma_{1}, \gamma_{2}$ sont des formes quadratiques d'Albert, alors les formes $\gamma_{1} \otimes \pi_{1}$ et $\gamma_{2} \otimes \pi_{2}$ sont semblables si et seulement si $\gamma_{1} \otimes \pi_{1} \perp \gamma_{2} \otimes \pi_{2} \in I^{k+3} F$ [6]. Mentionnons que ce résultat pour $k=1$ a été utilisé par Garibaldi [5, Theorem 15.4] dans la détermination de la forme de Killing de certains groupes de type $E_{8}$.

Dans ce papier, on va établir l'analogue de ce résultat en caractéristique 2 en prenant $\gamma_{1}$ et $\gamma_{2}$ des formes bilinéaires d'Albert, et $\pi_{1}$ et $\pi_{2}$ des formes quadratiques (ou bilinéaires) de Pfister, ou bien $\gamma_{1}$ et $\gamma_{2}$ des formes bilinéaires de Pfister et $\pi_{1}$ et $\pi_{2}$ des formes quadratiques d'Albert. Rappelons qu'en caractéristique 2, une forme bilinéaire d'Albert est une forme de dimension 6 et de déterminant trivial. Notre résultat principal est le théorème suivant :

ThÉORÈme 1. - Soit F un corps de caractéristique 2. Considérons les trois cas suivants :

(a) $\gamma_{1}, \gamma_{2}$ sont des formes bilinéaires d'Albert, et $\pi_{1}, \pi_{2}$ des $k$-formes quadratiques de Pfister, $k \geq 1$;

(b) $\gamma_{1}, \gamma_{2}$ sont des $k$-formes bilinéaires de Pfister, et $\pi_{1}, \pi_{2}$ des formes quadratiques d'Albert.

TOME $141-2013-\mathrm{N}^{\circ} 2$ 
(c) $\gamma_{1}, \gamma_{2}$ sont des formes bilinéaires d'Albert, et $\pi_{1}, \pi_{2}$ des $k$-formes bilinéaires de Pfister et les formes $\gamma_{i} \otimes \pi_{i}, i=1,2$, sont anisotropes.

Alors, les deux assertions suivantes sont équivalentes :

(1) $\gamma_{1} \otimes \pi_{1}$ est semblable $\grave{a} \gamma_{2} \otimes \pi_{2}$;

(2) $\gamma_{1} \otimes \pi_{1} \perp \gamma_{2} \otimes \pi_{2} \in I_{q}^{k+3} F$ dans les cas (a) et (b), et $\gamma_{1} \otimes \pi_{1} \perp \gamma_{2} \otimes \pi_{2} \in$ $I^{k+3} F$ dans le cas (c), respectivement.

Ce théorème dans le cas (a) est dû au second auteur lorsque $\pi_{1}, \pi_{2}$ sont des 0-formes bilinéaires de Pfister, i.e., des formes de dimension 1 données par : $(x, y) \mapsto x y[12]$.

La preuve du théorème 1 sera faite en trois étapes. Tout d'abord, on traitera le cas (a), c'est-à-dire, lorsque $\pi_{1}$ et $\pi_{2}$ sont des $k$-formes quadratiques de Pfister. Notre preuve dans ce cas est inspirée de l'argument utilisé dans [6]. Il s'en suit le cas (b) en invoquant des résultats connus sur la liaison des formes de Pfister en caractéristique 2. Après cela, on donnera la preuve dans le cas (c) en se ramenant au cas (a) par le moyen d'un argument générique. L'utilisation d'un tel argument est motivée par le fait que la preuve dans le cas quadratique ne marche pas dans le cas bilinéaire en raison du théorème de sous-forme qui se complique dans le cas des formes bilinéaires.

Pour la suite de ce papier, on suppose que $F$ est de caractéristique 2. En faisant l'analogie avec le théorème de Jacobson qui fait intervenir l'invariant de Clifford, on peut formuler le théorème 1 en termes d'invariants utilisant les formes différentielles. Rappelons que pour tout entier $n \geq 1$, on prend $\Omega_{F}^{n}=\wedge^{n} \Omega_{F}^{1}$ l'espace des $n$-formes différentielles $\left(\Omega_{F}^{0}=F\right)$, où $\Omega_{F}^{1}$ est le $F$-espace vectoriel engendré par les symboles $d x, x \in F$, avec les relations : $d(x+y)=d x+d y$ et $d(x y)=x d y+y d x$ pour tous $x, y \in F$. En particulier, $d\left(F^{2}\right)=0$, ce qui permet d'avoir une application $F^{2}$-linéaire $d: F \longrightarrow \Omega_{F}^{1}$, donnée par : $x \mapsto d x$. Cette application s'étend de manière naturelle en une application $d: \Omega_{F}^{n} \longrightarrow \Omega_{F}^{n+1}$, donnée par : $d\left(x d x_{1} \wedge \cdots \wedge d x_{n}\right)=d x \wedge d x_{1} \wedge$ $\cdots \wedge d x_{n}$. Clairement, l'espace $\Omega_{F}^{n}$ est engendré par les $n$-formes différentielles logarithmiques $\frac{d x_{1}}{x_{1}} \wedge \cdots \wedge \frac{d x_{n}}{x_{n}}$ pour $x_{1}, \cdots, x_{n} \in F^{*}:=F \backslash\{0\}$. L'opérateur d'Artin-Schreier s'étend en un homomorphisme $\wp: \Omega_{F}^{n} \longrightarrow \Omega_{F}^{n} / d \Omega_{F}^{n-1}$ donné par : $\wp\left(x \frac{d x_{1}}{x_{1}} \wedge \cdots \wedge \frac{d x_{n}}{x_{n}}\right)=\overline{\left(x^{2}-x\right) \frac{d x_{1}}{x_{1}} \wedge \cdots \wedge \frac{d x_{n}}{x_{n}}}$. Soient $\nu_{F}(n)=\operatorname{Ker}(\wp)$ et $H_{2}^{n+1}(F)=\operatorname{Coker}(\wp)$. Un résultat bien connu de Kato [9] affirme l'existence de deux isomorphismes $e^{n}: \overline{I^{n}} F \longrightarrow \nu_{F}(n)$ et $f^{n}: \overline{I_{q}^{n}} F \longrightarrow H_{2}^{n}(F)$ donnés sur les générateurs comme suit :

$$
e^{n}\left(\overline{\left\langle\left\langle a_{1}, \cdots, a_{n}\right\rangle\right\rangle_{b}}\right)=\frac{d a_{1}}{a_{1}} \wedge \cdots \wedge \frac{d a_{n}}{a_{n}},
$$




$$
f^{n}\left(\overline{\left\langle\left\langle a_{1}, \cdots, a_{n-1}, b\right]\right]}\right)=\overline{b \frac{d a_{1}}{a_{1}} \wedge \cdots \wedge \frac{d a_{n-1}}{a_{n-1}}}
$$

où $\left\langle\left\langle a_{1}, \cdots, a_{n}\right\rangle_{b}\right.$ est la $n$-forme bilinéaire de Pfister $\left\langle 1, a_{1}\right\rangle_{b} \otimes \cdots \otimes\left\langle 1, a_{n}\right\rangle_{b}$, sachant que $\left\langle c_{1}, \cdots, c_{n}\right\rangle_{b}$ désigne la forme bilinéaire diagonale $\sum_{i=1}^{n} c_{i} x_{i} y_{i}$ pour tous $c_{1}, \cdots, c_{n} \in F^{*}$, et $\left\langle\left\langle a_{1}, \cdots, a_{n-1}, b\right]\right]$ est la $n$-forme quadratique de Pfister $\left\langle\left\langle a_{1}, \cdots, a_{n-1}\right\rangle\right\rangle_{b} \otimes[1, b]$, où $[1, b]$ désigne la forme quadratique binaire $x^{2}+x y+$ $b y^{2}$.

Ainsi, aux formes $B \in I^{n} F$ et $\varphi \in I_{q}^{m} F$, on associe les formes différentielles

$$
\begin{gathered}
\operatorname{Inv}_{b}^{n}(B):=e^{n}\left(B+I^{n+1} F\right), \\
\operatorname{Inv}_{q}^{n+m}(B \otimes \varphi):=e^{n}\left(B+I^{n+1} F\right) \wedge f^{m}\left(\varphi+I_{q}^{m+1} F\right),
\end{gathered}
$$

qui sont des invariants de $B+I^{n+1} F \in \overline{I^{n}} F$ et $B \otimes \varphi+I_{q}^{n+m+1} F \in \overline{I_{q}^{n+m}} F$ par les isomorphismes ci-dessus. Par conséquent, le théorème 1 se reformule comme suit :

THÉORÈme 2. - En reprenant les notations du théorème 1, alors, les deux assertions suivantes sont équivalentes :

(1) $\gamma_{1} \otimes \pi_{1}$ est semblable à $\gamma_{2} \otimes \pi_{2}$;

(2) $\operatorname{Inv}_{q}^{k+2}\left(\gamma_{1} \otimes \pi_{1}\right)=\operatorname{Inv}_{q}^{k+2}\left(\gamma_{2} \otimes \pi_{2}\right)$ dans les cas (a) et (b), et $\operatorname{Inv}_{b}^{k+2}\left(\gamma_{1} \otimes\right.$ $\left.\pi_{1}\right)=\operatorname{Inv}_{b}^{k+2}\left(\gamma_{2} \otimes \pi_{2}\right)$ dans le cas (c), respectivement.

\section{Rappels et résultats préliminaires}

On renvoie à [1] et [7] pour plus de détails sur certaines notions qu'on va utiliser sur les formes bilinéaires et quadratiques. Rappelons tout de même que deux formes bilinéaires (ou quadratiques) $\varphi$ et $\psi$ sont dites semblables si $\varphi \simeq \alpha \psi$ pour $\alpha \in F^{*}$ convenable. Pour $\varphi$ une forme quadratique, on note $F(\varphi)$ le corps de fonctions de la quadrique projective d'équation $\varphi=0$. La dimension d'une forme quadratique (ou bilinéaire) $\varphi$ est notée $\operatorname{dim} \varphi$. Si $B$ est une forme bilinéaire d'espace sous-jacent $V$, on désigne par $\widetilde{B}$ la forme quadratique définie sur $V$ par : $\widetilde{B}(v)=B(v, v)$ pour $v \in V$. Pour $a_{1}, \cdots, a_{n} \in$ $F$, on note $\left\langle a_{1}, \cdots, a_{n}\right\rangle$ la forme quadratique diagonale $\sum_{i=1}^{n} a_{i} x_{i}^{2}$. Une forme quadratique (ou bilinéaire) $\varphi$ d'espace sous-jacent $V$ est dite isotrope s'il existe $v \in V$ non nul tel que $\varphi(v)=0$ (ou $\widetilde{\varphi}(v)=0)$. L'indice de Witt et la partie anisotrope d'une forme quadratique non singulière (ou bilinéaire) $\varphi$ sont notés $i_{W}(\varphi)$ et $\varphi_{\text {an }}$, respectivement. Un plan métabolique est une forme bilinéaire isotrope de dimension 2 ; on montre qu'une telle forme est donnée par la matrice $\mathbb{M}_{a}=\left(\begin{array}{ll}a & 1 \\ 1 & 0\end{array}\right)$ pour $a \in F$ convenable, et on a $\mathbb{M}_{a} \simeq \mathbb{M}_{b}$ ssi $a F^{* 2}=b F^{* 2}$, ce qui montre que pour les formes bilinéaires il y a plusieurs plans métaboliques à 
isométrie près. Si $B$ est une forme bilinéaire, on note $i_{d}(\widetilde{B})$ l'unique entier $n$ tel que $\operatorname{dim} B=n+\operatorname{dim}(\widetilde{B})_{\text {an }}$, qu'on appelle l'indice de défaut de $\widetilde{B}$. Rappelons la comparaison entre $i_{W}(B)$ et $i_{d}(\widetilde{B})$ :

Proposition 1. - Pour toute forme bilinéaire $B$, on a $i_{W}(B) \leq i_{d}(\widetilde{B}) \leq$ $2 i_{W}(B)$.

Preuve. D'après [14, Proposition 2.1], il existe deux entiers $r$ et $s$ uniques, et des scalaires $a_{1}, \cdots a_{s} \in F^{*}$ tels que

$$
B \simeq r \times \mathbb{M}_{0} \perp \mathbb{M}_{a_{1}} \perp \cdots \perp \mathbb{M}_{a_{s}} \perp B_{\mathrm{an}},
$$

avec la condition que la forme quadratique $\left\langle a_{1}, \cdots, a_{s}\right\rangle \perp \widetilde{\left(B_{\mathrm{an}}\right)}$ soit anisotrope. Donc, $i_{W}(B)=r+s$ et $\widetilde{B} \simeq(2 r+s) \times\langle 0\rangle \perp\left\langle a_{1}, \cdots, a_{s}\right\rangle \perp \widetilde{\left(B_{\text {an }}\right)}$. Par l'anisotropie de $\left\langle a_{1}, \cdots, a_{s}\right\rangle \perp \widetilde{\left(B_{\text {an }}\right)}$, on obtient $i_{d}(\widetilde{B})=2 r+s$. Ainsi, $i_{W}(B) \leq$ $i_{d}(\widetilde{B}) \leq 2 i_{W}(B)$.

Pour $\varphi$ une forme quadratique (ou bilinéaire) d'espace sous-jacent $V$, on note $D_{F}(\varphi)$ l'ensemble des scalaires non nuls représentés par $\varphi$ (ou $\widetilde{\varphi}$ ). Une forme quadratique (ou bilinéaire) de Pfister $\varphi$ vérifie la propriété de multiplicativité, ce qui signifie que $\varphi \simeq \alpha \varphi$ pour tout scalaire $\alpha \in D_{F}(\varphi)$; de plus, si $\varphi$ est isotrope, alors elle est hyperbolique (ou métabolique). Comme conséquence du théorème de sous-forme [7, Th. 4.2(iii)], si $\varphi$ est une forme quadratique de Pfister anisotrope et $\psi$ une forme quadratique anisotrope qui devient hyperbolique sur $F(\varphi)$, i.e. $i_{W}\left(\psi_{F(\varphi)}\right)=\frac{\operatorname{dim} \psi}{2}$, alors il existe une forme bilinéaire $B$ telle que $\psi \simeq B \otimes \varphi$, en particulier, $\operatorname{dim} \psi$ est un multiple de $\operatorname{dim} \varphi$. Concernant les formes quadratiques de $I_{q}^{n} F$, on aura besoin du résultat suivant :

Proposition 2. - (13, Prop. 6.4]) Soient $n \geq 1$ un entier et $\varphi$ est une forme quadratique anisotrope appartenant $\grave{a} I_{q}^{n} F$. Alors :

(1) $\operatorname{dim} \varphi=0$ ou $\operatorname{dim} \varphi \geq 2^{n}$ (c'est ce qu'on appelle le Hauptsatz "d'ArasonPfister"). De plus, si $\operatorname{dim} \varphi=2^{n}$, alors $\varphi$ est semblable à une $n$-forme quadratique de Pfister.

(2) Si, de plus, $\operatorname{dim} \varphi>2^{n}$, alors $\operatorname{dim} \varphi \geq 2^{n}+2^{n-1}$.

Le théorème suivant sera utilisé dans la preuve du théorème 1 dans le cas quadratique :

ThÉORÈme 3. - Soient $a, b, d \in F^{*}, \pi$ une $k$-forme quadratique de Pfister, et $\varphi$ une forme quadratique de dimension $2^{k+2}$. Alors, les assertions suivantes sont équivalentes :

(1) Il existe $c \in F^{*}$ tel que $\langle a, b, a b, d\rangle_{b} \otimes \pi \perp c \varphi \in I_{q}^{k+3} F$. 
(2) $\left(\langle a, b, a b, d\rangle_{b} \otimes \pi\right)_{\text {an }}$ est semblable à $\varphi_{\text {an }}$.

(3) $\langle a, b, a b, d\rangle_{b} \otimes \pi$ est semblable $\grave{a} \varphi$.

Preuve. $(2) \Longleftrightarrow(3)$ C'est une conséquence immédiate de l'équivalence de Witt pour les formes quadratiques.

$(2) \Longrightarrow(1)$ Cette implication est claire.

$(1) \Longrightarrow(2)$ On peut supposer que $c=1$. La forme $\rho:=\langle a, b, a b, d\rangle_{b} \otimes \pi \perp$ $\varphi \in I_{q}^{k+3} F$ est de dimension $2^{k+3}$. Si $\rho$ est isotrope, alors on obtient par le Hauptsatz que $\rho$ est hyperbolique, et donc $\left(\langle a, b, a b, d\rangle_{b} \otimes \pi\right)_{\text {an }} \simeq \varphi_{\text {an }}$. Ainsi, pour la suite, on peut supposer que $\rho$ est anisotrope.

(1) Supposons que $\pi \simeq d \pi$. Alors, $\langle a, b, a b, d\rangle_{b} \otimes \pi \simeq\langle\langle a, b\rangle\rangle_{b} \otimes \pi$. Soit $x \in$ $D_{F}(\varphi)$. On a que $\langle\langle a, b\rangle\rangle_{b} \otimes \pi \perp\langle x\rangle$ est une voisine anisotrope de $\langle\langle a, b, x\rangle\rangle_{b} \otimes \pi$, et par conséquent, $\langle\langle a, b, x\rangle\rangle_{b} \otimes \pi$ est aussi anisotrope (on renvoie à [7, Section 6] pour les détails sur les formes quadratiques voisines). On vérifie facilement que $\operatorname{dim}\left(\rho \perp\langle\langle a, b, x\rangle\rangle_{b} \otimes \pi\right)_{\text {an }} \leq 2^{k+3}-2$. Par le Hauptsatz, $\rho \perp\langle\langle a, b, x\rangle\rangle_{b} \otimes \pi \sim 0$. Par l'anisotropie de $\rho$ et $\langle\langle a, b, x\rangle\rangle_{b} \otimes \pi$, on déduit que $\rho \simeq\langle\langle a, b, x\rangle\rangle_{b} \otimes \pi$, ce qui donne que $\varphi \simeq x\langle a, b, a b, d\rangle_{b} \otimes \pi$.

(2) Supposons que $\pi \not 4 d \pi$. Remarquons d'abord que ceci implique que la forme de Pfister quadratique $\langle 1, d\rangle_{b} \otimes \pi$ est anisotrope. Comme $\langle a, b, a b\rangle_{b} \otimes \pi$ est une voisine anisotrope de $\langle\langle a, b\rangle\rangle_{b} \otimes \pi$, la forme $\langle\langle a, b\rangle\rangle_{b} \otimes \pi$ est aussi anisotrope. Soit $\psi:=\left(\rho \perp\langle\langle a, b\rangle\rangle_{b} \otimes \pi\right)_{\text {an }}$. On a $\operatorname{dim} \rho-\operatorname{dim}\left(\langle\langle a, b\rangle\rangle_{b} \otimes \pi\right)=2^{k+2} \leq \operatorname{dim} \psi \leq$ $2^{k+3}+2^{k+2}-2 \operatorname{dim}\left(\langle a, b, a b\rangle_{b} \otimes \pi\right)=2^{k+3}-2^{k+1}<2^{k+3}$. Mais les formes $\rho$ et $\langle\langle a, b\rangle\rangle_{b} \otimes \pi$ sont isotropes sur $K:=F\left(\langle\langle a, b\rangle\rangle_{b} \otimes \pi\right)$, et donc elles deviennent hyperboliques sur $K$ (l'hyperbolicité de $\rho_{K}$ découle du Hauptsatz). Ainsi, $\operatorname{dim} \psi$ est divisible par $\operatorname{dim}\left(\langle\langle a, b\rangle\rangle_{b} \otimes \pi\right)=2^{k+2}$. Par conséquent, $\operatorname{dim} \psi=2^{k+2}$ et $\rho \simeq \psi \perp\langle\langle a, b\rangle\rangle_{b} \otimes \pi$. Par le cas (1), il existe $x \in F^{*}$ tel que $\psi \simeq x\langle\langle a, b\rangle\rangle_{b} \otimes \pi$ et $\rho \simeq\langle\langle a, b, x\rangle\rangle_{b} \otimes \pi$.

Dans $W_{q}(F)$, on obtient $\langle\langle a, b, x\rangle\rangle_{b} \otimes \pi=\langle a, b, a b, d\rangle_{b} \otimes \pi \perp \varphi=\left\langle\langle a, b\rangle_{b} \otimes\right.$ $\pi \perp\langle 1, d\rangle_{b} \otimes \pi \perp \varphi$, et par conséquent $\varphi=x\langle\langle a, b\rangle\rangle_{b} \otimes \pi \perp\langle 1, d\rangle_{b} \otimes \pi$. Une comparaison des dimensions implique que $x\langle\langle a, b\rangle\rangle_{b} \otimes \pi \perp\langle 1, d\rangle_{b} \otimes \pi$ est isotrope. Comme $\langle\langle a, b\rangle\rangle_{b} \otimes \pi$ et $\langle 1, d\rangle_{b} \otimes \pi$ sont anisotropes, il existe $y \in D_{F}\left(x\langle\langle a, b\rangle\rangle_{b} \otimes \pi\right) \cap$ $D_{F}\left(\langle 1, d\rangle_{b} \otimes \pi\right)$. Par la multiplicativité d'une forme de Pfister, on a $x\langle\langle a, b\rangle\rangle_{b} \otimes$ $\pi \simeq y\langle\langle a, b\rangle\rangle_{b} \otimes \pi$, et $\langle 1, d\rangle_{b} \otimes \pi \simeq y\langle 1, d\rangle_{b} \otimes \pi$. Par conséquent, dans $W_{q}(F)$, on obtient $\varphi=y\left(\langle\langle a, b\rangle\rangle_{b} \otimes \pi \perp\langle 1, d\rangle_{b} \otimes \pi\right)=y\langle a, b, a b, d\rangle_{b} \otimes \pi$, et l'anisotropie de $\varphi$ et une comparaison des dimensions donne $\varphi \simeq y\langle a, b, a b, d\rangle_{b} \otimes \pi$.

On a aussi un analogue du théorème 3 pour les formes bilinéaires.

ThÉORÈme 4. - Soient $a, b, d \in F^{*}, \pi$ une $k$-forme bilinéaire de Pfister, et $\varphi$ une forme bilinéaire de dimension $2^{k+2}$. Alors, les assertions suivantes sont équivalentes : 
(1) Il existe $c \in F^{*}$ tel que $\langle a, b, a b, d\rangle_{b} \otimes \pi \perp c \varphi \in I^{k+3} F$.

(2) $\left(\langle a, b, a b, d\rangle_{b} \otimes \pi\right)_{\text {an }}$ est semblable $\grave{a} \varphi_{\text {an }}$.

Dans ce théorème on exclut la condition de similitude entre les formes bilinéaires elles-mêmes car, en général, deux formes bilinéaires de même dimension qui sont Witt-équivalentes ne sont pas nécesssairement isométriques. On démontre ce théorème de la même façon que le théorème 3. Les deux résultats essentiels dont on aura besoin sont : Le Hauptsatz pour les formes bilinéaires [11, Lemma 4.8], et le fait qu'une forme bilinéaire anisotrope qui devient métabolique sur $F(\widetilde{B})$, avec $B$ une $k$-forme bilinéaire de Pfister, est de dimension divisible par $2^{k}$ [10, Theorem 1.2].

\section{Preuve du théorème $1:$ Cas $(\mathrm{a})$}

Soient $\gamma_{1}, \gamma_{2}$ des formes bilinéaires d'Albert, et $\pi_{1}, \pi_{2}$ des $k$-formes quadratiques de Pfister, $k \geq 1$.

$(1) \Longrightarrow(2)$ Supposons que $\gamma_{1} \otimes \pi_{1}$ soit semblable à $\gamma_{2} \otimes \pi_{2}$. Soit $\alpha \in F^{*}$ tel que $\gamma_{1} \otimes \pi_{1} \simeq \alpha \gamma_{2} \otimes \pi_{2}$. Puisque $\langle 1, \alpha\rangle_{b} \otimes \gamma_{2} \in I^{3} F$, on déduit que $\gamma_{1} \otimes \pi_{1} \perp$ $\gamma_{2} \otimes \pi_{2} \in I_{q}^{k+3} F$.

$(2) \Longrightarrow(1)$ Supposons qu'on ait $\gamma_{1} \otimes \pi_{1} \perp \gamma_{2} \otimes \pi_{2} \in I_{q}^{k+3} F$.

$\mathrm{Si}$, disons, $\pi_{1}$ est isotrope et donc hyperbolique, alors $\gamma_{1} \otimes \pi_{1}$ est hyperbolique et $\gamma_{2} \otimes \pi_{2}$ l'est aussi par le Hauptsatz et l'implication est claire. Donc, on peut supposer que $\pi_{1}$ et $\pi_{2}$ sont anisotropes.

(i) Réduction au cas $\pi_{1} \simeq \pi_{2}$ : La forme $\left(\gamma_{1} \otimes \pi_{1}\right)_{F\left(\pi_{1}\right)}$ est hyperbolique, et par le Hauptsatz, la forme $\left(\gamma_{2} \otimes \pi_{2}\right)_{F\left(\pi_{1}\right)}$ l'est aussi. Posons $\psi \simeq\left(\gamma_{2} \otimes \pi_{2}\right)_{\text {an }}$. Ainsi, il existe une forme bilinéaire $\delta^{\prime}$ telle que $\psi \simeq \delta^{\prime} \otimes \pi_{1} \sim \gamma_{2} \otimes \pi_{2}$. Par la multiplicativité de $\pi_{1}$ (ou bien par le Hauptsatz), on voit que $\operatorname{dim} \delta^{\prime}$ doit être paire, donc en rajoutant des plans métaboliques $\langle 1,1\rangle_{b} \simeq \mathbb{M}_{1}$ à $\delta^{\prime}$, on conclut qu'il existe une forme bilinéaire $\delta$ de dimension 6 telle que $\gamma_{2} \otimes \pi_{2} \simeq$ $\delta \otimes \pi_{1}$. Soient $\lambda$ une forme de dimension 5 et $r \in F^{*}$ tels que $\delta \simeq \lambda \perp\langle r\rangle_{b}$. Puisque $\langle\operatorname{det} \lambda, r\rangle_{b} \otimes \pi_{1} \sim\left(\lambda \perp\langle\operatorname{det} \lambda\rangle_{b}\right) \otimes \pi_{1} \perp \delta \otimes \pi_{1}$, et que les formes $\left(\lambda \perp\langle\operatorname{det} \lambda\rangle_{b}\right) \otimes \pi_{1}$ et $\delta \otimes \pi_{1}$ appartiennent à $I_{q}^{k+2} F$, on déduit par le Hauptsatz que $\langle\text { det } \lambda, r\rangle_{b} \otimes \pi_{1} \sim 0$. Par l'unicité de la partie anisotrope, on a $\langle\operatorname{det} \lambda\rangle_{b} \otimes \pi_{1} \simeq$ $\langle r\rangle_{b} \otimes \pi_{1}$. Par conséquent, $\gamma_{2} \otimes \pi_{2} \simeq \delta \otimes \pi_{1} \simeq\left(\lambda \perp\langle\operatorname{det} \lambda\rangle_{b}\right) \otimes \pi_{1}$ avec $\lambda \perp\langle\operatorname{det} \lambda\rangle_{b}$ une forme bilinéaire d'Albert. Ainsi, sans perdre de généralité, on peut supposer que $\pi_{1} \simeq \pi_{2}$.

(ii) Montrons que $\gamma_{1} \otimes \pi_{1}$ est semblable à $\gamma_{2} \otimes \pi_{1}$ : Soient $\alpha \in D_{F}\left(\gamma_{1}\right)$ et $\beta \in D_{F}\left(\gamma_{2}\right)$. On pose $\gamma_{1}=\langle\alpha\rangle_{b} \perp \delta_{1}$ et $\gamma_{2}=\langle\beta\rangle_{b} \perp \delta_{2}$ avec $\operatorname{dim} \delta_{1}=$ 
$\operatorname{dim} \delta_{2}=5$. Puisque $\gamma_{1} \perp \alpha \gamma_{1} \in I^{3} F$ et $\gamma_{2} \perp \beta \gamma_{2} \in I^{3} F$, on déduit que $\left(\alpha \gamma_{1} \perp \beta \gamma_{2}\right) \otimes \pi_{1} \in I_{q}^{k+3} F$. En particulier,

$$
\left(\alpha \delta_{1} \perp \beta \delta_{2}\right) \otimes \pi_{1} \in I_{q}^{k+3} F .
$$

On a $\operatorname{dim}\left(\left(\alpha \delta_{1} \perp \beta \delta_{2}\right) \otimes \pi_{1}\right)_{\text {an }} \leq 10.2^{k}=2^{k+3}+2^{k+1}<2^{k+3}+2^{k+2}$. Par la proposition 2, $\operatorname{dim}\left(\left(\alpha \delta_{1} \perp \beta \delta_{2}\right) \otimes \pi_{1}\right)_{\text {an }} \leq 2^{k+3}$, en particulier, $\left(\alpha \delta_{1} \perp \beta \delta_{2}\right) \otimes \pi_{1}$ est isotrope. Par la multiplicativité de $\pi_{1}$, il existe $k \in F^{*}$, et $\lambda_{1}, \lambda_{2}$ des formes bilinéaires de dimension 4 tels que $\alpha \delta_{1} \otimes \pi_{1} \simeq\left(\langle k\rangle_{b} \perp \lambda_{1}\right) \otimes \pi_{1}$ et $\beta \delta_{2} \otimes \pi_{1} \simeq\left(\langle k\rangle_{b} \perp \lambda_{2}\right) \otimes \pi_{1}$. Ainsi, $\lambda_{1} \otimes \pi_{1} \perp \lambda_{2} \otimes \pi_{1} \in I_{q}^{k+3} F$. Par le théorème 3 , il existe $l \in F^{*}$ tel que $\lambda_{1} \otimes \pi_{1} \simeq l\left(\lambda_{2} \otimes \pi_{1}\right)$. En récapitulant les relations dès le début de $(\mathrm{a})$, on a :

$$
\begin{aligned}
\alpha\left(\gamma_{1} \otimes \pi_{1}\right) & =\pi_{1} \perp \alpha\left(\delta_{1} \otimes \pi_{1}\right) \\
& =\langle 1, k\rangle_{b} \otimes \pi_{1} \perp \lambda_{1} \otimes \pi_{1},
\end{aligned}
$$

et

$$
\begin{aligned}
l \beta\left(\gamma_{2} \otimes \pi_{1}\right) & =l \pi_{1} \perp l \beta\left(\delta_{2} \otimes \pi_{1}\right) \\
& =\langle l, k l\rangle_{b} \otimes \pi_{1} \perp l\left(\lambda_{2} \otimes \pi_{1}\right) .
\end{aligned}
$$

Comme $\alpha\left(\gamma_{1} \otimes \pi_{1}\right) \perp l \beta\left(\gamma_{2} \otimes \pi_{1}\right) \in I_{q}^{k+3} F$ et $\lambda_{1} \otimes \pi_{1} \simeq l\left(\lambda_{2} \otimes \pi_{1}\right)$, on déduit, par le Hauptsatz, que $\langle 1, k\rangle_{b} \otimes \pi_{1} \simeq\langle l, k l\rangle_{b} \otimes \pi_{1}$. Par conséquent, $\alpha\left(\gamma_{1} \otimes \pi_{1}\right) \simeq$ $l \beta\left(\gamma_{2} \otimes \pi_{1}\right)$.

\section{Preuve du théorème 1 : Cas $(b)$}

Pour traiter ce cas-là, il nous faut rappeler quelques résultats sur la liaison des formes de Pfister quadratiques en caractéristique 2 .

Soient $\pi_{1}$ et $\pi_{2}$ des $n$-formes quadratiques de Pfister, $n \geq 1$.

(i) Soit $0 \leq m<n$ un entier. On dit que $\pi_{1}$ et $\pi_{2}$ sont $m$-liées à gauche s'il existe une $m$-forme bilinéaire de Pfister $\beta$ et des $(n-m)$-formes quadratiques de Pfister $\rho_{1}, \rho_{2}$ telles que $\pi_{i} \simeq \beta \otimes \rho_{i}, i=1,2$.

(ii) Soit $0<m \leq n$ un entier. On dit que $\pi_{1}$ et $\pi_{2}$ sont $m$-liées à droite s'il existe une $m$-forme quadratique de Pfister $\gamma$ et des $(n-m)$-formes bilinéaires de Pfister $\sigma_{1}, \sigma_{2}$ telles que $\pi_{i} \simeq \sigma_{i} \otimes \gamma, i=1,2$.

Lemme 1. - Soient $\pi_{1}$ et $\pi_{2}$ des $n$-formes quadratiques de Pfister, $n \geq 1$.

(1) $i_{W}\left(\pi_{1} \perp \pi_{2}\right) \in\left\{1,2, \ldots, 2^{n}\right\}$, et $i_{W}\left(\pi_{1} \perp \pi_{2}\right) \geq 2^{m}, 0<m \leq n$, si et seulement si $\pi_{1}$ et $\pi_{2}$ sont $m$-liées à droite.

(2) Soit $0<m<n$. Si $\pi_{1}$ et $\pi_{2}$ sont $m$-liées à gauche, alors $\pi_{1}$ et $\pi_{2}$ sont $m$-liées à droite.

TOME $141-2013-\mathrm{N}^{\mathrm{O}} 2$ 
La première partie est due à Faivre [4, Théorème 2.3.1]. Une autre preuve se trouve dans $[3, \S 24]$. En ce qui concerne la deuxième partie, pour $n=2$, l'enoncé dit que si $\pi_{1} \simeq\left\langle\left\langle a, b_{1}\right]\right]$ et $\pi_{2} \simeq\left\langle\left\langle a, b_{2}\right]\right]$, alors il existe $c_{1}, c_{2} \in F^{*}$, $d \in F$ tels que $\pi_{1} \simeq\left\langle\left\langle c_{1}, d\right]\right]$ et $\pi_{2} \simeq\left\langle\left\langle c_{2}, d\right]\right]$. Dans ce cas, le résultat est dû à Draxl [2] et on sait que la réciproque est fausse en général, voir Baeza, [1, Remark 4.26, page 134]. Un aperçu de l'histoire de ce problème (avec une autre démonstration et un autre contre-exemple concernant la réciproque) se trouve dans Lam [15]. C'était Faivre [4, Corollaire 2.1.4] qui a remarqué que le cas général est une conséquence immédiate du cas $n=2$.

Il est clair que la preuve du cas (b) du théorème 1 se réduit au cas (a) qu'on vient de traiter en utilisant le lemme suivant.

Lemme 2. - Soient $\rho$ une $k$-forme bilinéaire de Pfister et $\alpha$ une forme quadratique d'Albert. Alors, il existe $\delta$ une forme bilinéaire d'Albert et $\gamma$ une $k$-forme quadratique de Pfister telles que $\rho \otimes \alpha \simeq \delta \otimes \gamma$.

Preuve. Puisque $\alpha$ est une forme quadratique d'Albert, on a $\operatorname{dim} \alpha=6$ et l'invariant d'Arf de $\alpha$ est trivial. Après multiplication par un scalaire on peut supposer

$$
\alpha \simeq c_{1}\left[1, x_{1}\right] \perp c_{2}\left[1, x_{2}\right] \perp\left[1, x_{1}+x_{2}\right] \sim\left\langle\left\langle c_{1}, x_{1}\right]\right] \perp\left\langle\left\langle c_{2}, x_{2}\right]\right] .
$$

En posant $\sigma_{i} \simeq \rho \otimes\left\langle\left\langle c_{i}, x_{i}\right]\right], i=1,2$, on constate que

$$
\rho \otimes \alpha \sim \sigma_{1} \perp \sigma_{2},
$$

avec $\sigma_{1}$ et $\sigma_{2}$ des $(k+2)$-formes quadratiques de Pfister qui sont $k$-liées à gauche, donc $k$-liées à droite, et par conséquent, il existe $r_{i}, s_{i} \in F^{*}, i=1,2$, et une $k$-forme quadratique de Pfister $\gamma$ tels que $\sigma_{i} \simeq\left\langle\left\langle r_{i}, s_{i}\right\rangle_{b} \otimes \gamma\right.$. Maintenant, on pose $\delta \simeq\left\langle r_{1}, s_{1}, r_{1} s_{1}, r_{2}, s_{2}, r_{2} s_{2}\right\rangle_{b}$ qui est une forme bilinéaire d'Albert et on obtient

$$
\rho \otimes \alpha \sim \sigma_{1} \perp \sigma_{2} \sim \delta \otimes \gamma
$$

et une comparaison des dimensions donne $\rho \otimes \alpha \simeq \delta \otimes \gamma$.

\section{Preuve du théorème $1:$ Cas (c)}

Soient $\gamma_{1}, \gamma_{2}$ des formes bilinéaires d'Albert, et $\pi_{1}, \pi_{2}$ des $k$-formes bilinéaires de Pfister. On suppose que les formes $\gamma_{1} \otimes \pi_{1}$ et $\gamma_{2} \otimes \pi_{2}$ sont anisotropes. Comme dans le cas quadratique, si la forme $\gamma_{1} \otimes \pi_{1}$ est semblable à $\gamma_{2} \otimes \pi_{2}$, alors $\gamma_{1} \otimes \pi_{1} \perp$ $\gamma_{2} \otimes \pi_{2} \in I^{k+3} F$. Réciproquement, supposons que $\gamma_{1} \otimes \pi_{1} \perp \gamma_{2} \otimes \pi_{2} \in I^{k+3} F$. Montrons que $\gamma_{1} \otimes \pi_{1}$ est semblable à $\gamma_{2} \otimes \pi_{2}$. Soit $K:=F((t))$ le corps des séries formelles en la variable $t$ sur $F$. La condition $\gamma_{1} \otimes \pi_{1} \perp \gamma_{2} \otimes \pi_{2} \in I^{k+3} F$ implique la suivante :

$$
\gamma_{1} \otimes \pi_{1} \otimes\left[1, t^{-1}\right] \perp \gamma_{2} \otimes \pi_{2} \otimes\left[1, t^{-1}\right] \in I_{q}^{k+4} K
$$


Les formes quadratiques $\gamma_{1} \otimes \pi_{1} \otimes\left[1, t^{-1}\right]$ et $\gamma_{2} \otimes \pi_{2} \otimes\left[1, t^{-1}\right]$ sont anisotropes [11, Lemme 4.6]. Par le cas quadratique, il existe $p \in K$ non nul tel que

$$
\gamma_{1} \otimes \pi_{1} \otimes\left[1, t^{-1}\right] \simeq p\left(\gamma_{2} \otimes \pi_{2} \otimes\left[1, t^{-1}\right]\right) .
$$

Sans perdre de généralité, on peut supposer que $p \in F[[t]]$. Puisque $t\left[1, t^{-1}\right] \simeq$ $\left[1, t^{-1}\right]$, on peut supposer que $p$ est une unité pour la valuation $t$-adique $v$ de $K$. En prenant dans la relation (1) la première forme résiduelle pour la valuation $v$, il existe $a \in F^{*}$ tel que $\widetilde{\gamma_{1}} \otimes \widetilde{\pi_{1}} \simeq a \widetilde{\gamma_{2}} \otimes \widetilde{\pi_{2}}$. Par conséquent, $i_{d}\left(\widetilde{\gamma_{1}} \otimes \widetilde{\pi_{1}} \perp\right.$ $\left.a \widetilde{\gamma_{2}} \otimes \widetilde{\pi_{2}}\right)=6.2^{k}$. Par la proposition 1 , on a $i_{W}\left(\gamma_{1} \otimes \pi_{1} \perp a \gamma_{2} \otimes \pi_{2}\right) \geq 3.2^{k}$, i.e., $\operatorname{dim}\left(\gamma_{1} \otimes \pi_{1} \perp a \gamma_{2} \otimes \pi_{2}\right)_{\text {an }} \leq 6.2^{k}$. Comme $\gamma_{1} \otimes \pi_{1} \perp \gamma_{2} \otimes \pi_{2} \equiv \gamma_{1} \otimes \pi_{1} \perp$ $a \gamma_{2} \otimes \pi_{2}\left(\bmod I^{k+3} F\right)$, on déduit, par le Hauptsatz pour les formes bilinéaires [11, Lemma 4.8], que $\gamma_{1} \otimes \pi_{1} \simeq a \gamma_{2} \otimes \pi_{2}$.

\section{Quelques commentaires}

La proposition suivante montre que le théorème 1 continue à être vrai même si dans le cas (c) on permet l'isotropie, pourvu que l'on considère les parties anisotropes des formes $\gamma_{i} \otimes \pi_{i}$.

Proposition 3. - On reprend les notations du théorème 1, mais on suppose maintenant que $\gamma_{1} \otimes \pi_{1}$ est isotrope. Alors $\gamma_{2} \otimes \pi_{2}$ est isotrope et $\left(\gamma_{1} \otimes \pi_{1}\right)$ an est semblable à $\left(\gamma_{2} \otimes \pi_{2}\right)$ an.

Preuve. D'abord, on a montré que les formes que l'on considère dans le cas (b) peuvent s'écrire comme des formes dans le cas (a). Ainsi, il suffit de traiter les cas (a) et (c). Donc, on peut supposer que $\gamma_{i}, i=1,2$ sont des formes bilinéaires d'Albert, et $\pi_{i}, i=1,2$ sont des $k$-formes quadratiques (cas (a)) resp. bilinéaires (cas (c)) de Pfister. La proposition 2 et l'isotropie de $\gamma_{1} \otimes \pi_{1}$ implique que $\operatorname{dim}\left(\gamma_{1} \otimes \pi_{1}\right)_{\text {an }} \in\left\{0,2^{k+2}\right\}$.

(i) Si $\operatorname{dim}\left(\gamma_{1} \otimes \pi_{1}\right)_{\text {an }}=0$, alors par le Hauptsatz $\operatorname{dim}\left(\gamma_{2} \otimes \pi_{2}\right)_{\text {an }}=0$ et il n'y a rien à montrer.

(ii) $\operatorname{Si} \operatorname{dim}\left(\gamma_{1} \otimes \pi_{1}\right)_{\text {an }}=2^{k+2}$, alors $\left(\gamma_{1} \otimes \pi_{1}\right)_{\text {an }} \simeq \delta \otimes \pi_{1}$ avec $\delta$ de dimension 4 et de déterminant trivial, en particulier, $\left(\gamma_{1} \otimes \pi_{1}\right)$ an est semblable à une $(k+2)$ forme de Pfister. On a aussi $\operatorname{dim}\left(\gamma_{2} \otimes \pi_{2}\right)$ an $>0$ par le Hauptsatz. Puisque $\left(\gamma_{1} \otimes \pi_{1}\right)$ an devient hyperbolique (ou métabolique) sur $K:=F\left(\left(\gamma_{1} \otimes \pi_{1}\right)\right.$ an $)$, on déduit par le Hauptsatz que $\gamma_{2} \otimes \pi_{2}$ est hyperbolique (ou métabolique) sur $K$. Ainsi, $\operatorname{dim}\left(\gamma_{2} \otimes \pi_{2}\right)$ an est un multiple de $\operatorname{dim}\left(\gamma_{1} \otimes \pi_{1}\right)_{\text {an }}=2^{k+2}$, voir [10], donc forcément $\operatorname{dim}\left(\gamma_{2} \otimes \pi_{2}\right)$ an $=2^{k+2}$, et en particulier $\gamma_{2} \otimes \pi_{2}$ est isotrope. Par les théorèmes 3 et 4 , la forme $\left(\gamma_{1} \otimes \pi_{1}\right)$ an est semblable à $\left(\gamma_{2} \otimes \pi_{2}\right)$ an . 
Dans le cas bilinéaire (cas (c)), le théorème 1 cesse d'être vrai si la forme bilinéaire $\gamma_{1} \otimes \pi_{1}$ ou $\gamma_{2} \otimes \pi_{2}$ est isotrope. En voici un exemple.

EXemple. - Soit $K=F\left(x, y, z, t, x_{1}, \cdots, x_{k}\right)$ le corps des fractions rationnelles en les variables $x, y, z, t, x_{1}, \cdots, x_{k}$ sur $F$. Soit $\pi=\left\langle\left\langle x_{1}, \cdots, x_{k}\right\rangle_{b}\right.$, $\gamma_{1}=\mathbb{M}_{0} \perp\langle\langle x, y\rangle\rangle_{b}$ et $\gamma_{2}=\mathbb{M}_{t} \perp z\langle\langle x, y\rangle\rangle_{b}$. Alors, $\gamma_{1} \otimes \pi \perp \gamma_{2} \otimes \pi \in I^{k+3} K$, mais $\gamma_{1} \otimes \pi$ n'est pas semblable à $\gamma_{2} \otimes \pi$.

Preuve. Puisque $\gamma_{1} \perp \gamma_{2} \in I^{3} K$, on a $\gamma_{1} \otimes \pi \perp \gamma_{2} \otimes \pi \in I^{k+3} K$. Supposons que $\gamma_{1} \otimes \pi$ soit semblable à $\gamma_{2} \otimes \pi$ sur $K$. Soit $p \in K$ non nul tel que

$$
\gamma_{1} \otimes \pi \simeq p\left(\gamma_{2} \otimes \pi\right) .
$$

Puisque $\mathbb{M}_{t} \simeq\langle t, t\rangle_{b}$ et $\alpha \mathbb{M}_{0} \simeq \mathbb{M}_{0}$ pour tout $\alpha \in K^{*}$, la relation (2) devient

$$
2^{k} \times \mathbb{M}_{0} \perp\left\langle\langle x , y \rangle _ { b } \otimes \pi \simeq p \left(\langle t, t\rangle_{b} \otimes \pi \perp z\left\langle\langle x, y\rangle_{b} \otimes \pi\right) .\right.\right.
$$

En prenant la forme quadratique diagonale associée à la forme bilinéaire dans (3), on obtient

$$
2^{k+1} \times\langle 0\rangle \perp\langle 1, x, y, x y\rangle \otimes \tilde{\pi} \simeq p(\langle t, t\rangle \otimes \tilde{\pi} \perp z\langle 1, x, y, x y\rangle \otimes \tilde{\pi}),
$$

ce qui donne une contradiction puisque l'indice de défaut du membre de gauche est $2^{k+1}$, alors que celui du membre de droite est $2^{k}$.

\section{BIBLIOGRAPHIE}

[1] R. BAEZA - Quadratic forms over semilocal rings, Lecture Notes in Math., vol. 655, Springer, 1978.

[2] P. DRAXL - « Über gemeinsame separabel-quadratische Zerfällungskörper von Quaternionenalgebren », Nachr. Akad. Wiss. Göttingen Math.-Phys. Kl. II 16 (1975), p. 251-259.

[3] R. Elman, N. Karpenko \& A. MerkurJev - The algebraic and geometric theory of quadratic forms, American Mathematical Society Colloquium Publications, vol. 56, Amer. Math. Soc., 2008.

[4] F. FAIVRE - «Liaison des formes de Pfister et corps de fonctions de quadriques en caractéristique $2 »$, thèse de doctorat, Université de FrancheComté, 2006.

[5] S. GARIBALDi - «Orthogonal involutions on algebras of degree 16 and the Killing form of $E_{8} »$, in Quadratic forms-algebra, arithmetic, and geometry, Contemp. Math., vol. 493, Amer. Math. Soc., 2009, p. 131-162.

[6] D. W. Hoffmann - « On a conjecture of Izhboldin on similarity of quadratic forms », Doc. Math. 4 (1999), p. 61-64. 
[7] D. W. Hoffmann \& A. Laghribi - « Quadratic forms and Pfister neighbors in characteristic $2 »$, Trans. Amer. Math. Soc. 356 (2004), p. 40194053.

[8] N. JACOBSON - « Some applications of Jordan norms to involutorial simple associative algebras », Adv. in Math. 48 (1983), p. 149-165.

[9] K. KATO - « Symmetric bilinear forms, quadratic forms and Milnor $K$ theory in characteristic two », Invent. Math. 66 (1982), p. 493-510.

[10] A. LAGHRIBI - «Witt kernels of function field extensions in characteristic $2 »$, J. Pure Appl. Algebra 199 (2005), p. 167-182.

[11] _ « Sur le déploiement des formes bilinéaires en caractéristique $2 »$, Pacific J. Math. 232 (2007), p. 207-232.

[12] « Jacobson's theorem for bilinear forms in characteristic 2 », Proc. Amer. Math. Soc. 137 (2009), p. 2905-2912.

[13] _ « Les formes bilinéaires et quadratiques bonnes de hauteur 2 en caractéristique $2 »$, Math. Z. 269 (2011), p. 671-685.

[14] A. Laghribi \& P. Mammone - «Hyper-isotropy of bilinear forms in characteristic 2 », in Quadratic forms-algebra, arithmetic, and geometry, Contemp. Math., vol. 493, Amer. Math. Soc., 2009, p. 249-269.

[15] T. Y. LAM - « On the linkage of quaternion algebras », Bull. Belg. Math. Soc. Simon Stevin 9 (2002), p. 415-418.

[16] P. Mammone \& D. B. Shapiro - « The Albert quadratic form for an algebra of degree four », Proc. Amer. Math. Soc. 105 (1989), p. 525-530.

[17] A. S. MERKUR'Ev - « On the norm residue symbol of degree $2 »$, Dokl. Akad. Nauk SSSR 261 (1981), p. 542-547.

[18] C. H. SAH - «Symmetric bilinear forms and quadratic forms », J. Algebra 20 (1972), p. 144-160. 